\title{
Effect of Magnetic Field on Reflection of Thermo-elastic Waves from the Boundary of a Half-Space Using G-N Model of Type-II for Different Nature of the Boundary Surface
}

\author{
Manik Chandra Singh ${ }^{1} \cdot$ Nilratan Chakraborty $^{2}$
}

Published online: 12 August 2015

(C) Springer India Pvt. Ltd. 2015

\begin{abstract}
The aim of this paper is to investigate the problem of reflection of plane thermoelastic waves in presence of magnetic field from the boundary of a half-space using Green-Naghdi model of type-II. Analytical solutions for the ratios of the amplitude of the reflected waves to that of incident wave have been obtained in matrix form for stress-free insulated, stress-free isothermal, rigid insulated and rigid isothermal boundaries of the halfspace. Numerical results have been carried out to obtain the values the amplitude ratios only for incident $\mathrm{P}$ and SV wave and shown graphically.
\end{abstract}

Keywords Reflection - Generalized thermoelasticity · Magnetic field · G-N model of type-II

\section{Introduction}

The classical theory of thermo-elasticity based on parabolic heat conduction equation given by Biot [1] predicts infinite speed of propagation for thermal signals. It leads to the physically unrealistic phenomenon when applied to problems such as those dealing with sudden heat inputs or extremely low temperature regimes. To eliminate this paradox inherent in the classical theory, non-classical or generalized theories which admit finite speed of propagation for thermal signals have been developed in the last five decades. Chronologically, Lord and Shulmon [2] formulated the extended thermo-elasticity theory (ETE) by incorporating a flux-rate term into the conventional Fourier's law of heat conduction and introduced a single time constant to dictate the relaxation of thermal propagation. Subsequently Green and Lindsay [3]

\footnotetext{
Manik Chandra Singh

maniksingh1981@gmail.com

Nilratan Chakraborty

nil_c53@yahoo.co.in

1 Balarampur P. C. H. S. School, Rangadih (P.O), Purulia (Dist.), West Bengal 723143, India

2 University Department of Physics, Kolhan University, Chaibasa, Jharkhand 833201, India
} 
developed temperature-rate-dependent thermo-elasticity (TRDTE) theory by introducing two relaxation times and thus heat propagation was conceptualized as a wave phenomenon rather than a diffusion one. Chandrasekharaiah [4] referred wave-like thermal disturbances as "second sound". Most of the metals are not suitable for use in experiments concerning second sound propagation as they possess a relatively high rate of thermal damping. Green and Naghdi [5-7] developed three models (type- I, II and III) of generalized thermoelasticity which provide the relevant theoretical development to identify or even manufacture for laboratory purposes an idealized material for the purpose of studying the propagation of thermal waves at finite speed. Among these three models of G-N theory, the most significant fact is that the internal rate of production of entropy in type-II is identically zero implying no dissipation of thermal energy. The G-N model of type-II admits undamped thermoelastic waves in a thermoelastic material and is known as the theory of thermoelasticity without energy dissipation.

Considerable research articles on reflection or propagation of plane thermoelastic waves have been reported by many researchers such as Sinha and Elsibai [8], Sharma et al. [9], Othman and Song [10], Chakraborty and Singh [11], Singh and Chakraborty [12] using various models of generalized thermoelasticity under different physical conditions. But in these papers the presence of magnetic field has not been considered.

Magnetic field plays very important and significant role in seismic wave propagation, damping of acoustic waves, plasma and many more and during the last two decades, many researchers contributed to the development of Magneto-thermoelasticity. Knopoff [13], Chadwick [14] and Nowacki [15] studied the interaction between magnetic field and strain field at the beginning. The problem of magneto-thermo-elastic plane wave was first studied by Paria [16]. Later problems on propagation of magneto-thermo-elastic waves were studied by Roychoudhuri and Banerjee [17] and Singh [18] using L-S model. Considering G-N model of type-II, Roychoudhuri and Banerjee [19] studied the magneto-elastic plane waves in rotating media and Othman and Song [20] considered the effect of rotation on the reflection of magneto-thermoelastic waves. Abo-Dahab and Salama [21] discussed the reflection and refraction of plane magneto-thermoelastic waves at solid-solid interface in presence of external heat sources and initial stress. Recently, reflection of plane magneto-thermo-elastic waves in presence of initial stress has been studied by Singh and Chakraborty [22].

In this paper the effects of magnetic field on reflection of thermoelastic waves under different thermo-mechanical conditions of the boundary of a solid half-space have been investigated using G-N model of type-II.

\section{Formulation of the Problem}

We consider a linear, homogeneous, isotropic thermoelastic solid half-space at uniform absolute temperature $\mathrm{T}_{0}$ in the undisturbed state. We assume that the half-space under consideration is a perfect electric conductor and permeated by a magnetic field. When a plane wave (P or SV or thermal) is incident at the boundary of the half-space, we get reflected Pwave, SV-wave and thermal wave in the solid medium M (Fig. 1). We would like to calculate the ratios of amplitudes of the three reflected components to that of the incident wave for different values of magnetic field.

We assume a fixed rectangular Cartesian coordinate system oxyz with origin 'o' on the boundary of the thermo-elastic solid half-space at $y=0$ and negative $y$-axis pointing normally into the solid medium, which we identify as region y $<0$. The magnetic field $\vec{H}_{0}=(0,0, H)$ 


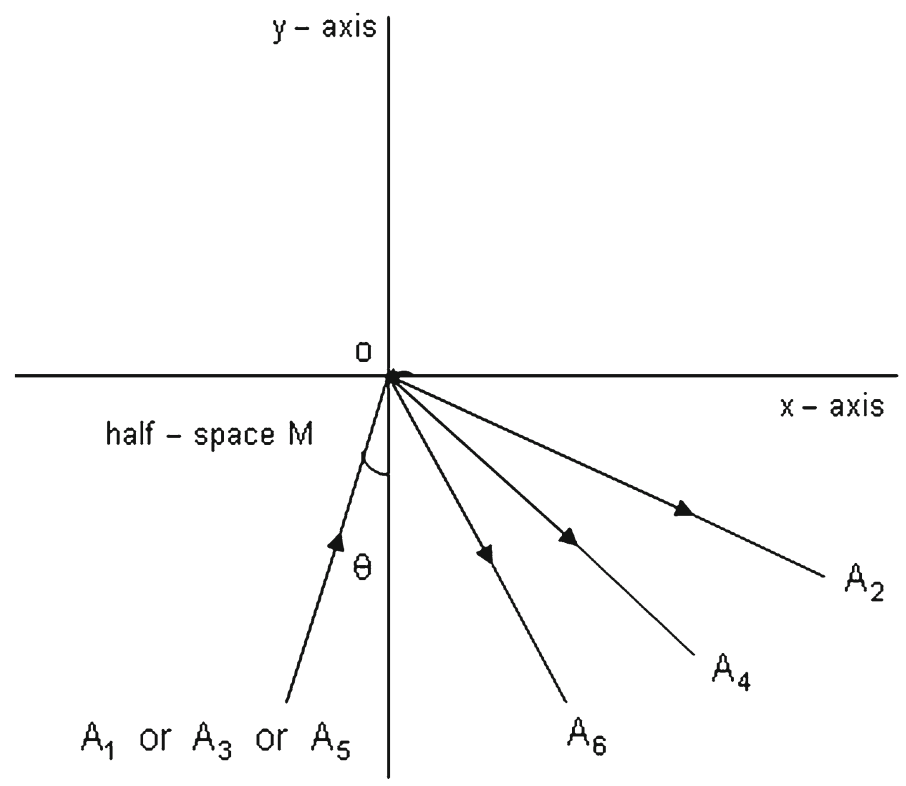

Fig. 1 Geometry of the problem

is acting along the z-axis. Since we consider a two-dimensional problem, we restrict our analysis to plane strain parallel to oxy-plane. Hence all the field variables depend only on space coordinates $\mathrm{x}, \mathrm{y}$ besides time $\mathrm{t}$ and are independent of coordinate $\mathrm{z}$.

\section{Basic Equations}

(1) The dynamical equations of motion for plane strain in absence of heat source and initial stress, given by Biot [1], taking into account the presence of Lorentz force are

$$
\begin{aligned}
& \frac{\partial s_{11}}{\partial x}+\frac{\partial s_{12}}{\partial y}+F_{1}=\rho \frac{\partial^{2} u}{\partial t^{2}}, \\
& \frac{\partial s_{12}}{\partial x}+\frac{\partial s_{22}}{\partial y}+F_{2}=\rho \frac{\partial^{2} v}{\partial t^{2}},
\end{aligned}
$$

where $\mathrm{s}_{11}, \mathrm{~s}_{22}, \mathrm{~s}_{12}$ are incremental stress components, produced due to deformation. The first two are principal stress components and the last one is shear stress component. $\mathrm{F}_{1}$ and $\mathrm{u}$ are the components Lorentz force and displacement vector along $\mathrm{x}$-axis respectively while $\mathrm{F}_{2}$ and $\mathrm{v}$ are the similar quantities along $\mathrm{y}$-axis.

(2) The stress-strain relations with incremental isotropy are given by Biot [1] as

$$
\mathrm{s}_{11}=(\lambda+2 \mu) \mathrm{e}_{\mathrm{xx}}+\lambda \mathrm{e}_{\mathrm{yy}}-\gamma \mathrm{T}, \mathrm{s}_{22}=\lambda \mathrm{e}_{\mathrm{xx}}+(\lambda+2 \mu) \mathrm{e}_{\mathrm{yy}}-\gamma \mathrm{T}, \mathrm{s}_{12}=2 \mu \mathrm{e}_{\mathrm{xy}},
$$

where $e_{x x}, e_{y y}$ are the principal strain components, $e_{x y}$ is the shear strain component, $\lambda$ and $\mu$ are Lame's constants, $\gamma=(3 \lambda+2 \mu) \alpha_{t}, \alpha_{t}$ is the coefficient of linear thermal expansion and $\mathrm{T}$ is the incremental change in temperature.

(3) The incremental strain components are given by Biot [1] as

$$
e_{x x}=\frac{\partial u}{\partial x}, e_{y y}=\frac{\partial v}{\partial y}, e_{x y}=\frac{1}{2}\left(\frac{\partial v}{\partial x}+\frac{\partial u}{\partial y}\right) .
$$


(4) The modified heat conduction equation for G-N theory of type-II is

$$
\kappa^{*} \nabla^{2} \mathrm{~T}=\rho \mathrm{C}_{\mathrm{e}} \frac{\partial^{2} \mathrm{~T}}{\partial \mathrm{t}^{2}}+\mathrm{T}_{0} \gamma \frac{\partial^{2}}{\partial \mathrm{t}^{2}}\left(\frac{\partial \mathrm{u}}{\partial \mathrm{x}}+\frac{\partial \mathrm{v}}{\partial \mathrm{y}}\right)
$$

where $\rho, \mathrm{C}_{\mathrm{e}}$ and $\mathrm{T}_{0}$ are the density, specific heat per unit mass at constant strain and initial uniform temperature in absolute scale of the elastic medium respectively and $\kappa^{*}=\mathrm{C}_{\mathrm{e}}(\lambda+2 \mu) / 4$ is a characteristic constant of the G-N theory of type-II.

(5) Taking into account the absence of displacement current, the linearized Maxwell equations governing the electromagnetic fields for a slowly moving solid medium having perfect electrical conductivity are

$$
\begin{aligned}
\operatorname{curl} \overrightarrow{\mathrm{h}}=\overrightarrow{\mathrm{J}}, \operatorname{curl} \overrightarrow{\mathrm{E}} & =-\mu_{\mathrm{e}} \frac{\partial \overrightarrow{\mathrm{h}}}{\partial \mathrm{t}}, \quad \operatorname{div} \overrightarrow{\mathrm{h}}=0, \operatorname{div} \overrightarrow{\mathrm{E}}=0, \\
\text { where } \overrightarrow{\mathrm{h}} & =\operatorname{curl}\left(\overrightarrow{\mathrm{u}} \times \overrightarrow{\mathrm{H}}_{0}\right) .
\end{aligned}
$$

$\vec{E}, \vec{J}, \vec{H}_{0}, \mu_{e}, \vec{h}$ and $\vec{u}(u, v, 0)$ are the electrical intensity, electric current density, primary magnetic field, magnetic permeability, perturbed magnetic field over the constant primary magnetic field vector and the displacement vector respectively.

Evaluating $\overrightarrow{\mathrm{J}}$ of Eq. (5) with the help of Eq. (6) and then putting the value of $\overrightarrow{\mathrm{J}}$ in the equation of Lorentz force $\overrightarrow{\mathrm{F}}=\mu_{\mathrm{e}}\left(\overrightarrow{\mathrm{J}} \times \overrightarrow{\mathrm{H}}_{0}\right)$, we get the components of Lorentz force as

$$
F_{1}=\mu_{e} H^{2}\left(\frac{\partial^{2} u}{\partial x^{2}}+\frac{\partial^{2} v}{\partial x \partial y}\right) \text { and } F_{2}=\mu_{e} H^{2}\left(\frac{\partial^{2} u}{\partial x \partial y}+\frac{\partial^{2} v}{\partial y^{2}}\right)
$$

Again Maxwell's stress equation can be given in the form as

$$
\tau_{i j}=\mu_{e}\left[H_{i} h_{j}+H_{j} h_{i}-(\vec{H} \cdot \vec{h}) \delta_{i j}\right], \quad(\text { where } i, j=1,2,3) .
$$

Equation (8) gives

$$
\tau_{22}=\mu_{\mathrm{e}} \mathrm{H}^{2}\left(\frac{\partial \mathrm{u}}{\partial \mathrm{x}}+\frac{\partial \mathrm{v}}{\partial \mathrm{y}}\right) \text { and } \tau_{12}=0 .
$$

\section{Solution of the Problem}

Making use of Eqs. (2), (3) and (7) in Eq. (1), we obtain,

$$
\begin{aligned}
& \left(\lambda+2 \mu+\mu_{\mathrm{e}} \mathrm{H}^{2}\right) \frac{\partial^{2} \mathrm{u}}{\partial \mathrm{x}^{2}}+\left(\lambda+\mu+\mu_{\mathrm{e}} \mathrm{H}^{2}\right) \frac{\partial^{2} \mathrm{v}}{\partial \mathrm{x} \partial \mathrm{y}}+\mu \frac{\partial^{2} \mathrm{u}}{\partial \mathrm{y}^{2}}=\rho \frac{\partial^{2} \mathrm{u}}{\partial \mathrm{t}^{2}}+\gamma \frac{\partial \mathrm{T}}{\partial \mathrm{x}} \\
& \left(\lambda+2 \mu+\mu_{\mathrm{e}} \mathrm{H}^{2}\right) \frac{\partial^{2} \mathrm{v}}{\partial \mathrm{y}^{2}}+\left(\lambda+\mu+\mu_{\mathrm{e}} \mathrm{H}^{2}\right) \frac{\partial^{2} \mathrm{u}}{\partial \mathrm{x} \partial \mathrm{y}}+\mu \frac{\partial^{2} \mathrm{v}}{\partial \mathrm{x}^{2}}=\rho \frac{\partial^{2} \mathrm{v}}{\partial \mathrm{t}^{2}}+\gamma \frac{\partial \mathrm{T}}{\partial \mathrm{y}}
\end{aligned}
$$

To separate the dilatational and rotational components of strain, we introduce displacement potentials $\phi$ and $\psi$ defined by the following relations:

$$
\mathrm{u}=\frac{\partial \phi}{\partial \mathrm{x}}-\frac{\partial \psi}{\partial \mathrm{y}} \quad \text { and } \mathrm{v}=\frac{\partial \phi}{\partial \mathrm{y}}+\frac{\partial \psi}{\partial \mathrm{x}} .
$$


Using Eqs. (11) in (10a) and then integrating partially with respect to variables $\mathrm{x}$ and $\mathrm{y}$, we get,

$$
\begin{gathered}
\nabla^{2} \phi=\frac{\rho}{\left(\lambda+2 \mu+\mu_{\mathrm{e}} \mathrm{H}^{2}\right)} \frac{\partial^{2} \phi}{\partial \mathrm{t}^{2}}+\frac{\gamma}{\left(\lambda+2 \mu+\mu_{\mathrm{e}} \mathrm{H}^{2}\right)} \mathrm{T}, \\
\nabla^{2} \psi=\frac{\rho}{\mu} \frac{\partial^{2} \psi}{\partial \mathrm{t}^{2}} .
\end{gathered}
$$

Proceeding exactly in a similar way by putting Eqs. (11) in (10b), and then partially integrating with respect to $y$ and $x$ respectively, we get,

$$
\begin{gathered}
\nabla^{2} \phi=\frac{\rho}{\left(\lambda+2 \mu+\mu_{\mathrm{e}} \mathrm{H}^{2}\right)} \frac{\partial^{2} \phi}{\partial \mathrm{t}^{2}}+\frac{\gamma}{\left(\lambda+2 \mu+\mu_{\mathrm{e}} \mathrm{H}^{2}\right)} \mathrm{T}, \\
\nabla^{2} \psi=\frac{\rho}{\mu} \frac{\partial^{2} \psi}{\partial \mathrm{t}^{2}},
\end{gathered}
$$

where $\nabla^{2}=\frac{\partial^{2}}{\partial \mathrm{x}^{2}}+\frac{\partial^{2}}{\partial \mathrm{y}^{2}}$.

Therefore Eqs. (12a) and (12c) involving scalar potential $\phi$ represent dilatational waves along $\mathrm{x}$ - and $\mathrm{y}$-axis respectively while Eqs. (12d) and (12b) involving vector potential $\psi$, represent rotational waves along $\mathrm{x}$ - and $\mathrm{y}$-axis respectively. Since we consider dilatational and rotational waves propagating along $\mathrm{x}$-axis, we take Eqs. (12a) and (12d) and they can be rewritten as,

$$
\begin{gathered}
\nabla^{2} \phi=\frac{1}{\mathrm{c}_{1}^{2}\left(1+\mathrm{R}_{\mathrm{H}}\right)} \frac{\partial^{2} \phi}{\partial \mathrm{t}^{2}}+\frac{\gamma}{\rho \mathrm{c}_{1}{ }^{2}\left(1+\mathrm{R}_{\mathrm{H}}\right)} \mathrm{T}, \\
\text { and } \nabla^{2} \psi=\frac{1}{\mathrm{c}_{2}^{2}} \frac{\partial^{2} \psi}{\partial \mathrm{t}^{2}} .
\end{gathered}
$$

where $\mathrm{R}_{\mathrm{H}}=\frac{\mathrm{c}_{\mathrm{A}}^{2}}{\mathrm{c}_{1}^{2}}, \mathrm{c}_{\mathrm{A}}^{2}=\frac{\mu_{\mathrm{e}} \mathrm{H}^{2}}{\rho}, \mathrm{c}_{1}^{2}=\frac{\lambda+2 \mu}{\rho}, \mathrm{c}_{2}^{2}=\frac{\mu}{\rho}$. Here $\mathrm{R}_{\mathrm{H}}, \mathrm{c}_{\mathrm{A}}, \mathrm{c}_{1}, \mathrm{c}_{2}$ represent magnetic pressure number, Alfven wave velocity, isothermal dilatational and rotational wave velocity respectively.

Using Eqs. (11) in (4), we get,

$$
\kappa^{*} \nabla^{2} \mathrm{~T}=\rho \mathrm{C}_{\mathrm{e}} \frac{\partial^{2} \mathrm{~T}}{\partial \mathrm{t}^{2}}+\mathrm{T}_{0} \gamma \frac{\partial^{2}}{\partial \mathrm{t}^{2}}\left(\nabla^{2} \phi\right) .
$$

Solving Eqs. (13a) and (14) simultaneously to eliminate ' $T$ ', the following 4th order differential equation involving scalar potential $\phi$ evolves

$$
\left(1+\mathrm{R}_{\mathrm{H}}\right) \mathrm{c}_{3}^{2} \nabla^{4} \phi-\left(1+\mathrm{R}_{\mathrm{H}}+\frac{\mathrm{c}_{3}^{2}}{\mathrm{c}_{1}^{2}}+\varepsilon_{\mathrm{T}}\right) \frac{\partial^{2}}{\partial \mathrm{t}^{2}}\left(\nabla^{2} \phi\right)+\frac{1}{\mathrm{c}_{1}^{2}} \frac{\partial^{4} \phi}{\partial \mathrm{t}^{4}}=0
$$

where $c_{3}^{2}=\frac{\kappa^{*}}{\rho c_{\mathrm{e}}}$ and $\varepsilon_{\mathrm{T}}=\frac{\mathrm{T}_{0} \gamma^{2}}{\rho^{2} \mathrm{C}_{\mathrm{e}} \mathrm{c}_{1}^{2}}$, is the thermoelastic coupling constant of the solid medium $\mathrm{M}$.

We write the trial solutions for $\phi, \psi$ and $\mathrm{T}$ as

$$
\begin{aligned}
\phi & =f(y) \exp [i k(x-c t)], \\
\psi & =g(y) \exp [i k(x-c t)], \\
T & =h(y) \exp [i k(x-c t)],
\end{aligned}
$$


where $\mathrm{c}=\omega / \mathrm{k}$ is the phase velocity, $\omega$ is the circular frequency and $\mathrm{k}$ is the wave number. Satisfying Eqs. (16a) in (15), we obtain,

$$
\begin{aligned}
& \left(1+R_{H}\right) \frac{d^{4} f}{d y^{4}}+\left[-2\left(1+R_{H}\right) k^{2}+\frac{k^{2} c^{2}}{c_{3}^{2}}\left(1+R_{H}+\varepsilon_{T}+\frac{c_{3}^{2}}{c_{1}^{2}}\right)\right] \frac{d^{2} f}{d y^{2}} \\
& +\left[k^{4}\left(1+R_{H}\right)-\frac{k^{4} c^{2}}{c_{3}^{2}}\left(1+R_{H}+\varepsilon_{T}+\frac{c_{3}^{2}}{c_{1}^{2}}\right)+\frac{k^{4} c^{4}}{c_{1}^{2} c_{3}^{2}}\right] f=0 .
\end{aligned}
$$

Equation (17) being a fourth order differential equation in $\mathrm{f}(\mathrm{y})$, the solution gives four values of $\mathrm{f}(\mathrm{y})$ and Eq. (16a) becomes

$$
\phi=\left[\begin{array}{r}
A_{1} \exp \left(i k m_{1} y\right)+A_{2} \exp \left(-i k m_{1} y\right) \\
+A_{3}\left(i k m_{2} y\right)+A_{4} \exp \left(-i k m_{2} y\right)
\end{array}\right] \exp [i k(x-c t)],
$$

where

$$
\begin{aligned}
\mathrm{m}_{1}= & \sqrt{\mathrm{q}^{2} \mathrm{c}^{2}-1}, \mathrm{~m}_{2}=\sqrt{\mathrm{p}^{2} \mathrm{c}^{2}-1} \\
\mathrm{p}^{2}, \mathrm{q}^{2}= & \frac{1}{2 \mathrm{c}_{1}^{2} \mathrm{c}_{3}^{2}\left(1+\mathrm{R}_{\mathrm{H}}\right)}\left[\mathrm{N} \pm \sqrt{\mathrm{N}^{2}-4\left(1+\mathrm{R}_{\mathrm{H}}\right) \mathrm{c}_{1}^{2} \mathrm{c}_{3}^{2}}\right] \\
& \text { and } \mathrm{N}=\left[\left(1+\mathrm{R}_{\mathrm{H}}+\varepsilon_{\mathrm{T}}\right) \mathrm{c}_{1}^{2}+\mathrm{c}_{3}^{2}\right] .
\end{aligned}
$$

Using Eqs. (16b) in (13b), we get,

$$
\frac{\mathrm{d}^{2} \mathrm{~g}}{\mathrm{dy}^{2}}+\mathrm{k}^{2}\left(\frac{\mathrm{c}^{2}}{\mathrm{c}_{2}^{2}}-1\right) \mathrm{g}=0
$$

Equation (20) suggests that the solution yields two values of $\mathrm{g}(\mathrm{y})$, and Eq. (16b) can be written as

$$
\begin{gathered}
\psi=\left[\mathrm{A}_{5} \exp \left(\mathrm{ikm}_{3} \mathrm{y}\right)+\mathrm{A}_{6} \exp \left(-\mathrm{ikm}_{3} \mathrm{y}\right)\right] \exp [\mathrm{ik}(\mathrm{x}-\mathrm{ct})] \\
\text { where } \mathrm{m}_{3}=\sqrt{\left(\mathrm{c}^{2} / \mathrm{c}_{2}^{2}\right)-1} .
\end{gathered}
$$

The constants $\mathrm{A}_{\mathrm{i}}(\mathrm{i}=1,2,3,4,5,6)$ in pairs represent the amplitudes of the incident and reflected thermal, $\mathrm{P}$ - and $\mathrm{SV}$-waves respectively.

Using Eqs. (16c) and (18) in Eq. (13a), we get the value of $\mathrm{h}(\mathrm{y})$ and the corresponding expression for $\mathrm{h}(\mathrm{y})$ is then put into Eq. (16c) to get

$$
\begin{aligned}
& \mathrm{T}=\frac{\rho}{\gamma}\left[\begin{array}{c}
\mathrm{b}_{1}\left\{\mathrm{~A}_{1} \exp \left(\mathrm{ikm}_{1} \mathrm{y}\right)+\mathrm{A}_{2} \exp \left(-\mathrm{ikm} \mathrm{m}_{1} \mathrm{y}\right)\right\} \\
+\mathrm{b}_{2}\left\{\mathrm{~A}_{3} \exp \left(\mathrm{ikm} \mathrm{ikm}_{2} \mathrm{y}\right)+\mathrm{A}_{4} \exp \left(-\mathrm{ikm} \mathrm{k}_{2} \mathrm{y}\right)\right\}
\end{array}\right] \exp [\mathrm{ik}(\mathrm{x}-\mathrm{ct})], \\
& \text { where } \mathrm{b}_{1}=\omega^{2}\left\{1-\left(1+\mathrm{R}_{\mathrm{H}}\right) \mathrm{q}^{2} \mathrm{c}_{1}^{2}\right\} \text { and } \mathrm{b}_{2}=\omega^{2}\left\{1-\left(1+\mathrm{R}_{\mathrm{H}}\right) \mathrm{p}^{2} \mathrm{c}_{1}^{2}\right\} .
\end{aligned}
$$

\section{Boundary Conditions}

Reflection of thermoelastic waves depends on the thermo-mechanical nature of the boundary of the given half-space and in this section a brief account of the mechanical and thermal boundary conditions have been included. 


\section{Mechanical Boundary Conditions}

Depending upon the mechanical conditions, boundary of a half-space may be stress-free or rigid.

\section{Stress-Free Boundary}

Since boundary is stress-free,

(1) The normal force per unit initial area must vanish at the boundary i.e. $\nabla f_{y}=0$ leads to $\mathrm{s}_{22}+\tau_{22}=0$.

Using Eqs. (2), (3), (9), (11), (18), (21) and (23) in the above equation, we get,

$$
\left(-2+\frac{c^{2}}{c_{2}^{2}}\right)\left(A_{1}+A_{2}\right)+\left(-2+\frac{c^{2}}{c_{2}^{2}}\right)\left(A_{3}+A_{4}\right)+2 m_{3}\left(A_{5}-A_{6}\right)=0,
$$

(2) The tangential force per unit initial area must vanish at the boundary i.e. $\nabla f_{x}=0$, leads to $\left(\mathrm{s}_{12}+\mathrm{Pe}_{\mathrm{x} y}\right)+\tau_{12}=0$.

Substituting Eqs. (2), (3), (9), (11), (18), (21) and (23) in the above equation, we get,

$$
m_{1}\left(A_{1}-A_{2}\right)+m_{2}\left(A_{3}-A_{4}\right)-\frac{1}{2}\left(m_{3}^{2}-1\right)\left(A_{5}+A_{6}\right)=0 .
$$

\section{Rigid Boundary}

Since boundary is rigid,

(1) The normal displacement must vanish at the boundary, i.e. $\mathrm{v}=0$.

Making use of Eqs. (11), (18) and (21) in the above equation, we obtain,

$$
m_{1}\left(A_{1}-A_{2}\right)+m_{2}\left(A_{3}-A_{4}\right)+A_{5}+A_{6}=0
$$

(2) The tangential displacement must vanish at the boundary, i.e. $\mathrm{u}=0$.

Using Eqs. (11), (18) and (21) in the above equation, we get,

$$
A_{1}+A_{2}+A_{3}+A_{4}-m_{3}\left(A_{5}-A_{6}\right)=0
$$

\section{Thermal Boundary Conditions}

Under different thermal conditions, we have either insulated or isothermal boundary.

\section{Insulated Boundary}

For a thermally insulated boundary, we have $\frac{\partial T}{\partial y}=0$.

Using Eq. (23) in the above equation and simplifying we get,

$$
m_{1}\left\{1-\left(1+R_{H}\right) q^{2} c_{1}^{2}\right\}\left(A_{1}-A_{2}\right)+m_{2}\left\{1-\left(1+R_{H}\right) p^{2} c_{1}^{2}\right\}\left(A_{3}-A_{4}\right)=0
$$




\section{Isothermal Boundary}

For an isothermal boundary, we have $\mathrm{T}=0$.

Using Eq. (23) in the above equation, we get,

$$
\left\{1-\left(1+R_{H}\right) q^{2} c_{1}^{2}\right\}\left(A_{1}+A_{2}\right)+\left\{1-\left(1+R_{H}\right) p^{2} c_{1}^{2}\right\}\left(A_{3}+A_{4}\right)=0
$$

\section{Equations for the Reflection Coefficients}

We develop the theory for a half-space with four different types of boundaries, namely stress-free insulated, stress-free isothermal, rigid insulated and rigid isothermal and to satisfy the thermo-mechanical conditions for each type of these boundaries, we have taken three boundary equations (two for mechanical and one for thermal condition) from the previous section. Since the plane wave incident at the boundary of the half-space making an angle $\theta$ with the $\mathrm{y}$-axis (Fig. 1) is either P-wave or thermal wave or SV-wave, the following substitutions are made to have the generalized expression of the boundary equations for each of the plane incident wave:

(a) For P-wave incidence: $\mathrm{c}=\mathrm{p}^{-1} \operatorname{cosec} \theta, \mathrm{A}_{1}=\mathrm{A}_{5}=0$ and $\mathrm{z}_{1}=\frac{\mathrm{A}_{2}}{\mathrm{~A}_{3}}, \mathrm{z}_{2}=\frac{\mathrm{A}_{4}}{\mathrm{~A}_{3}}, \mathrm{z}_{3}=\frac{\mathrm{A}_{6}}{\mathrm{~A}_{3}}$.

(b) For thermal wave incidence: $c=q^{-1} \operatorname{cosec} \theta, A_{3}=A_{5}=0$ and $z_{1}=\frac{A_{2}}{A_{1}}, z_{2}=$ $\frac{\mathrm{A}_{4}}{\mathrm{~A}_{1}}, \mathrm{z}_{3}=\frac{\mathrm{A}_{6}}{\mathrm{~A}_{1}}$.

(c) For SV-wave incidence: $c=c_{2} \operatorname{cosec} \theta, A_{1}=A_{3}=0$ and $z_{1}=\frac{A_{2}}{A_{5}}, z_{2}=\frac{A_{4}}{A_{5}}, z_{3}=\frac{A_{6}}{A_{5}}$.

Generalizing these three boundary equations, we get a system of three equations for a thermoelastic plane incident wave as:

$$
\sum_{j=1}^{3} a_{i j} z_{j}=y_{i}, \quad(\text { where } i=1,2,3),
$$

where $\mathrm{z}_{\mathrm{j}}(\mathrm{j}=1,2,3)$ are the ratios of amplitude of reflected thermal, $\mathrm{P}$ - and $\mathrm{SV}$-wave to that of the incident wave respectively and $\mathrm{a}_{\mathrm{ij}}$ are their coefficients.

Equations (31) constitute a matrix equation as

$$
\begin{gathered}
A Z=Y, \\
\text { Where } A=\left[\begin{array}{lll}
a_{11} & a_{12} & a_{13} \\
a_{21} & a_{22} & a_{23} \\
a_{31} & a_{32} & a_{33}
\end{array}\right], Z=\left[\begin{array}{l}
z_{1} \\
z_{2} \\
z_{3}
\end{array}\right], Y=\left[\begin{array}{l}
y_{1} \\
y_{2} \\
y_{3}
\end{array}\right] .
\end{gathered}
$$

Case 1 For Stress-free insulated boundary:

$\mathrm{a}_{11}=\left(-2+\frac{\mathrm{c}^{2}}{\mathrm{c}_{2}^{2}}\right), \quad \mathrm{a}_{12}=\left(-2+\frac{\mathrm{c}^{2}}{\mathrm{c}_{2}^{2}}\right), \quad \mathrm{a}_{13}=-2 \mathrm{~m}_{3}, \quad \mathrm{a}_{21}=-\mathrm{m}_{1}, \quad \mathrm{a}_{22}=-\mathrm{m}_{2}$,

$\mathrm{a}_{23}=-\left(\mathrm{m}_{3}^{2}-1\right) / 2, \mathrm{a}_{31}=-\mathrm{m}_{1}\left\{1-\left(1+\mathrm{R}_{\mathrm{H}}\right) \mathrm{q}^{2} \mathrm{c}_{1}^{2}\right\}, \quad \mathrm{a}_{32}=-\mathrm{m}_{2}\left\{1-\left(1+\mathrm{R}_{\mathrm{H}}\right) \mathrm{p}^{2} \mathrm{c}_{1}^{2}\right\}$, $\mathrm{a}_{33}=0$ and

(a) For P-wave incidence: $y_{1}=-a_{12}, y_{2}=a_{22}, y_{3}=a_{32}$.

(b) For SV-wave incidence: $y_{1}=a_{13}, y_{2}=-a_{23}, y_{3}=a_{33}$.

(c) For thermal wave incidence: $\mathrm{y}_{1}=-\mathrm{a}_{11}, \mathrm{y}_{2}=\mathrm{a}_{21}, \mathrm{y}_{3}=\mathrm{a}_{31}$. 
Case 2 For Stress-free isothermal boundary:

$\mathrm{a}_{11}=\left(-2+\frac{\mathrm{c}^{2}}{\mathrm{c}_{2}^{2}}\right), \quad \mathrm{a}_{12}=\left(-2+\frac{\mathrm{c}^{2}}{\mathrm{c}_{2}^{2}}\right), \quad \mathrm{a}_{13}=-2 \mathrm{~m}_{3}, \quad \mathrm{a}_{21}=-\mathrm{m}_{1}, \quad \mathrm{a}_{22}=-\mathrm{m}_{2}$,
$\mathrm{a}_{23}=-0.5\left(\mathrm{~m}_{3}^{2}-1\right), \quad \mathrm{a}_{31}=\left\{1-\left(1+\mathrm{R}_{\mathrm{H}}\right) \mathrm{q}^{2} \mathrm{c}_{1}^{2}\right\}, \quad \mathrm{a}_{32}=\left\{1-\left(1+\mathrm{R}_{\mathrm{H}}\right) \mathrm{p}^{2} \mathrm{c}_{1}^{2}\right\}, \quad \mathrm{a}_{33}=0$

and

(a) For P-wave incidence: $\mathrm{y}_{1}=-\mathrm{a}_{12}, \mathrm{y}_{2}=\mathrm{a}_{22}, \mathrm{y}_{3}=-\mathrm{a}_{32}$.

(b) For SV-wave incidence: $y_{1}=a_{13}, y_{2}=-a_{23}, y_{3}=a_{33}$.

(c) For thermal wave incidence: $\mathrm{y}_{1}=-\mathrm{a}_{11}, \mathrm{y}_{2}=\mathrm{a}_{21}, \mathrm{y}_{3}=-\mathrm{a}_{31}$.

Case 3 For Rigid insulated boundary:

$\mathrm{a}_{11}=-\mathrm{m}_{1}, \quad \mathrm{a}_{12}=-\mathrm{m}_{2}, \quad \mathrm{a}_{13}=1, \quad \mathrm{a}_{21}=1, \quad \mathrm{a}_{22}=1, \quad \mathrm{a}_{23}=\mathrm{m}_{3}$,

$\mathrm{a}_{31}=-\mathrm{m}_{1}\left\{1-\left(1+\mathrm{R}_{\mathrm{H}}\right) \mathrm{q}^{2} \mathrm{c}_{1}^{2}\right\}, \quad \mathrm{a}_{32}=-\mathrm{m}_{2}\left\{1-\left(1+\mathrm{R}_{\mathrm{H}}\right) \mathrm{p}^{2} \mathrm{c}_{1}^{2}\right\}, \quad \mathrm{a}_{33}=0$ and

(a) For P-wave incidence: $y_{1}=a_{12}, y_{2}=-a_{22}, y_{3}=a_{32}$.

(b) For SV-wave incidence: $y_{1}=-a_{13}, y_{2}=a_{23}, y_{3}=a_{33}$.

(c) For thermal wave incidence: $y_{1}=a_{11}, y_{2}=-a_{21}, y_{3}=a_{31}$.

Case 4 For Rigid isothermal boundary:

$a_{11}=-m_{1}, \quad a_{12}=-m_{2}, \quad a_{13}=1, \quad a_{21}=1, \quad a_{22}=1, \quad a_{23}=m_{3}, \quad a_{31}=\left\{1-\left(1+R_{H}\right) q^{2} c_{1}^{2}\right\}$,

$\mathrm{a}_{32}=\left\{1-\left(1+\mathrm{R}_{\mathrm{H}}\right) \mathrm{p}^{2} \mathrm{c}_{1}^{2}\right\}, \quad \mathrm{a}_{33}=0$ and

(a) For P-wave incidence: $y_{1}=a_{12}, y_{2}=-a_{22}, y_{3}=-a_{32}$.

(b) For SV-wave incidence: $\mathrm{y}_{1}=-\mathrm{a}_{13}, \mathrm{y}_{2}=\mathrm{a}_{23}, \mathrm{y}_{3}=\mathrm{a}_{33}$.

(c) For thermal wave incidence: $y_{1}=-a_{11}, y_{2}=-a_{21}, y_{3}=-a_{31}$.

\section{Numerical Results and Discussion}

For numerical analysis of the expressions derived in the previous section, we have used aluminium as solid medium.

For solid medium $\mathrm{M}: \lambda=57.75 \times 10^{9} \mathrm{Nm}^{-2}, \mu=26.43 \times 10^{9} \mathrm{Nm}^{-2}, \alpha=23 \times$ $10^{-6} \mathrm{k}^{-1}, \mathrm{C}_{\mathrm{e}}=900 \mathrm{~J} \mathrm{Kg}^{-1} \mathrm{~K}^{-1}, \rho=2700 \mathrm{Kg} \mathrm{m}^{-3}, \kappa=237 \mathrm{~W} \mathrm{~m}^{-1} \mathrm{~K}^{-1}$.

We have chosen the angular frequency of thermoelastic wave $\omega=7.5 \times 10^{11} \mathrm{~s}^{-1}$ and initial uniform temperature $\mathrm{T}_{0}=300 \mathrm{~K}$.

Moreover to show the variation of the amplitude ratios with angle of incidence for different values of magnetic field in the solid medium, we use magnetic pressure number $\left(\mathrm{R}_{\mathrm{H}}\right)$.

To solve the matrix Eq. (32), a computer programme, using MATLAB Software, has been developed and ratios of the amplitudes of the reflected waves to that of incident wave for different angle of incidence have been calculated. Numerical computations have been done for the following four types of boundary of a half-space.

\section{Effect of Magnetic Field for Stress-Free Insulated Boundary}

For P-wave incidence (Fig. 2a), magnetic field has little impact on $\left|\mathrm{z}_{1}\right|$ versus $\theta$ curve for $35^{\circ}>\theta>85^{\circ}$. For SV-wave incidence (Fig. 2b), magnetic field decreases the value of the first maximum of $\left|\mathrm{z}_{1}\right|$ and has no effect on $\left|\mathrm{z}_{1}\right|$ at $\theta=45^{\circ}$ and afterwards. 

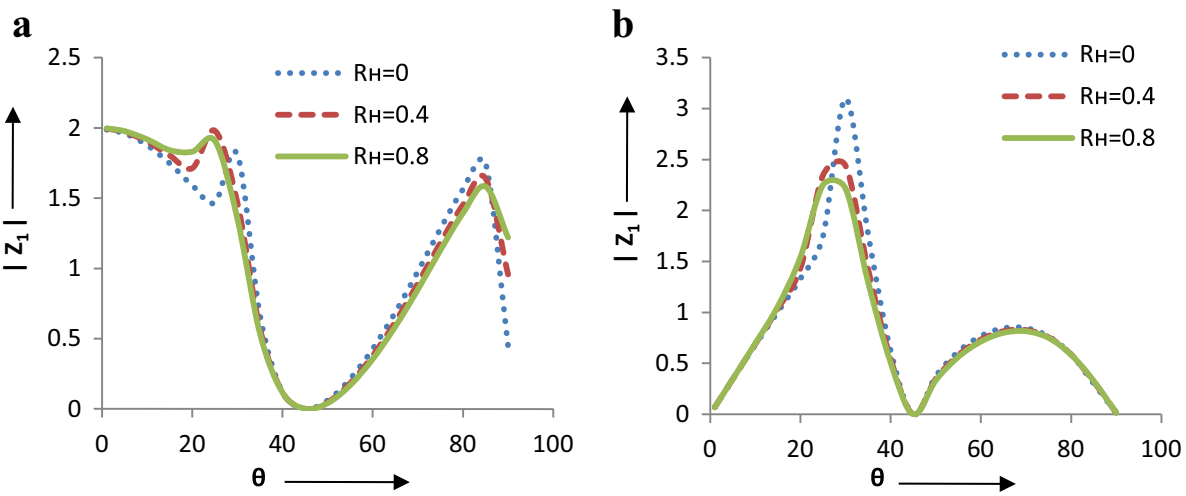

Fig. 2 Variation of the modulus of amplitude ratio of reflected thermal wave $\left[Z_{1}\right]$ from stress-free insulated boundary with angle of incidence $(\theta)$ for different values magnetic field for a P-wave incidence and $\mathbf{b} \mathrm{SV}$-wave incidence
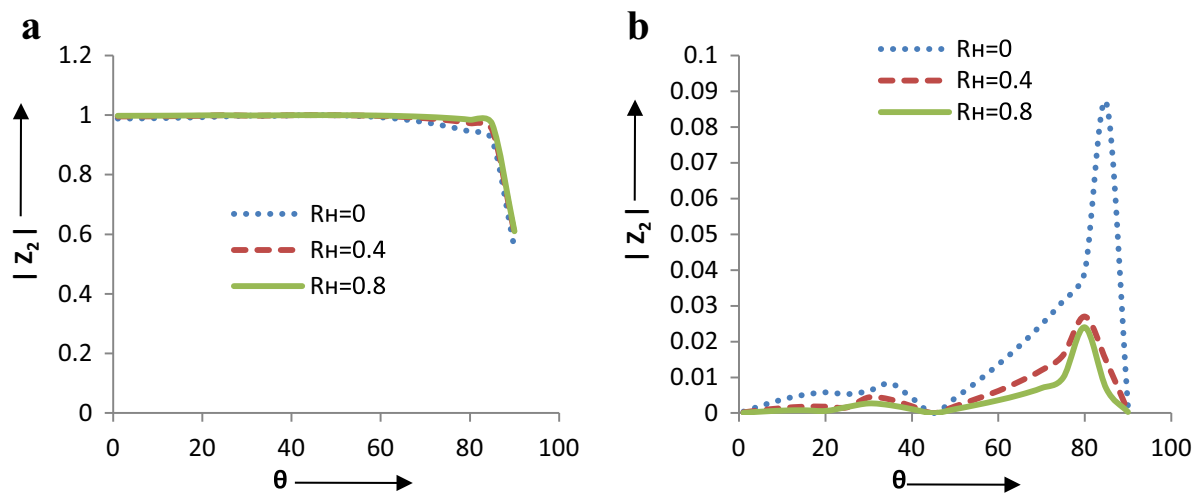

Fig. 3 Variation of the modulus of amplitude ratio of reflected $\mathrm{P}$-wave $\left[\mathrm{Z}_{2}\right]$ from stress-free insulated boundary with angle of incidence $(\theta)$ for different values magnetic field for $\mathbf{a} \mathrm{P}$-wave incidence and $\mathbf{b} \mathrm{SV}$-wave incidence

Figure 3a shows that magnetic field can not affect the value of $\left|z_{2}\right|$ for P-wave incidence whereas for SV-wave incidence (Fig. 3b) it can decrease the value of $\left|\mathrm{z}_{2}\right|$ and shift the peak from $\theta=85^{\circ}$ to $\theta=80^{\circ}$ with a lower magnitude.

For P-wave incidence (Fig. 4a), magnetic field can change the value of $\left|z_{3}\right|$ within $0^{\circ}<\theta<35^{\circ}$ and $85^{\circ}<\theta<90^{\circ}$, but for $35^{\circ}<\theta<85^{\circ},\left|z_{3}\right|$ decreases to zero at $\theta=45^{\circ}$ and sharply increases to its maximum value at $\theta=85^{\circ}$ showing its unchanged nature on the application of magnetic field. From Fig. 4b, it is noticed that for SV-wave incidence, $\left|z_{3}\right|$ decreases from unity to a minimum value, then increases to its maximum, and remains constant afterwards. Larger the magnetic field, smaller the curve dip.

\section{Effect of Magnetic Field for Stress-Free Isothermal Boundary}

It is noticed from Fig. 5a that the effect of magnetic field on $\left|z_{1}\right|$ versus $\theta$ curve is only to decrease its peak at $\theta=30^{\circ}$ for P-wave incidence. For incident SV-wave, variation of $\left|\mathrm{z}_{1}\right|$ (Fig. 5b) shows that the magnetic field can decrease the value its first maxima at $\theta=30^{\circ}$ but the second one at $\theta=70^{\circ}$ remains unchanged. 
$\mathbf{a}$

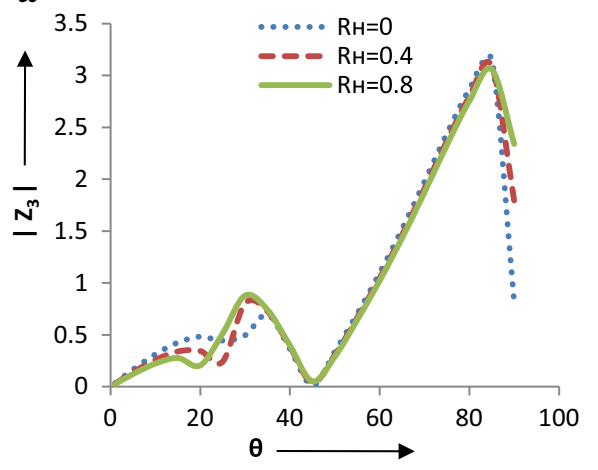

b

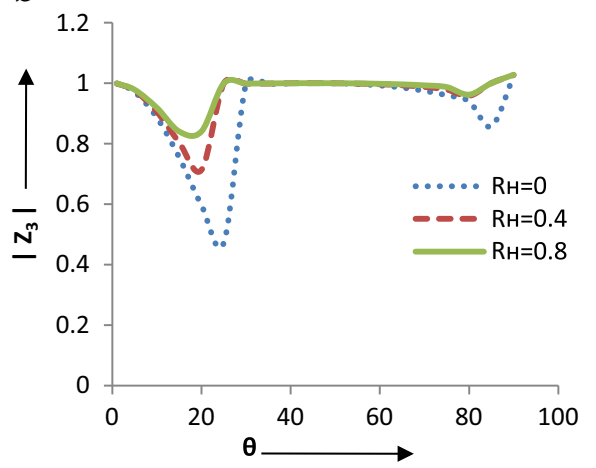

Fig. 4 Variation of the modulus of amplitude ratio of reflected SV-wave [ $Z_{3}$ ] from stress-free insulated boundary with angle of incidence $(\theta)$ for different values magnetic field for a P-wave incidence and $\mathbf{b}$ SVwave incidence

a

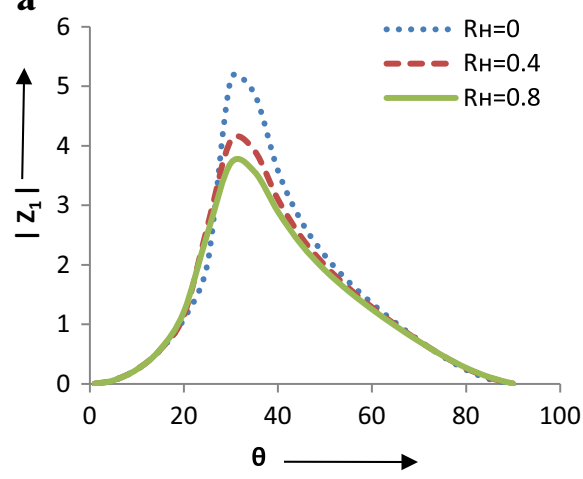

b

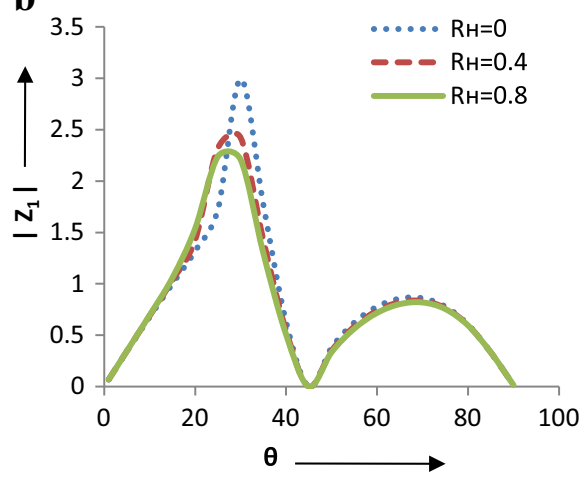

Fig. 5 Variation of the modulus of amplitude ratio of reflected thermal wave $\left[Z_{1}\right]$ from stress-free isothermal boundary with angle of incidence $(\theta)$ for different values magnetic field for $\mathbf{a} \mathrm{P}$-wave incidence and $\mathbf{b} \mathrm{SV}$-wave incidence

In Fig. 6a, $\left|z_{2}\right|$ decreases from its maximum value of unity, reaches minimum at $\theta=30^{\circ}$, sharply increases to one at $\theta=40^{\circ}$ and then remains almost constant afterwards, showing that the magnetic field can reduce the dip at $\theta=30^{\circ}$ for P-wave incidence. For SV-wave incidence, $\left|z_{2}\right|$ (Fig. 6b) shows very low values on either sides of its minimum at $\theta=45^{\circ}$ and for both the sides higher is magnetic field, lower is the value of $\left|z_{2}\right|$.

On increasing the magnetic field, the maximum value of the prominent peak of $\left|z_{3}\right|$ for incident $\mathrm{P}$-wave at $\theta=30^{\circ}$ decreases, but the other maximum at $\theta=70^{\circ}$ remains more or less same (Fig. 7a). For SV-wave incidence, with the application of the magnetic field, the dip of the curve of $\left|z_{3}\right|$ (Fig. 7a) decreases and the minimum of the curve appears at a lower value of $\theta$.

\section{Effect of Magnetic Field for Rigid Insulated Boundary}

Figure 8a shows that magnetic field can affect the $\left|\mathrm{z}_{1}\right|$ versus $\theta$ curve of P-wave incidence for $15^{\circ}\left\langle\theta\left\langle 50^{\circ}\right.\right.$ and $\theta>85^{\circ}$ and in between these two ranges the curve remains unchanged. 

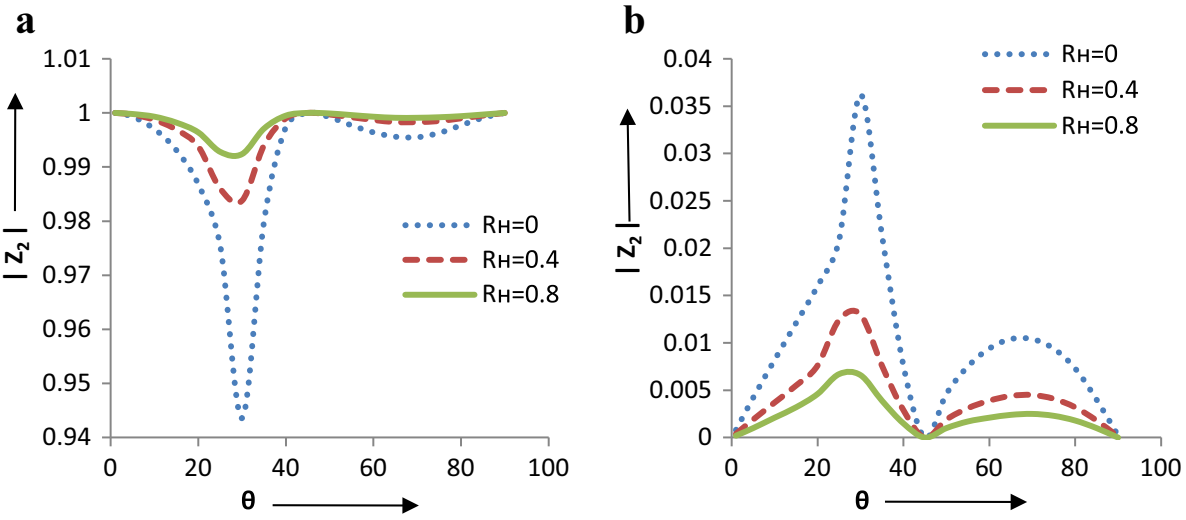

Fig. 6 Variation of the modulus of amplitude ratio of reflected P-wave [ $\mathrm{Z}_{2}$ ] from stress-free isothermal boundary with angle of incidence $(\theta)$ for different values magnetic field for a P-wave incidence and $\mathbf{b} \mathrm{SV}$ wave incidence

a

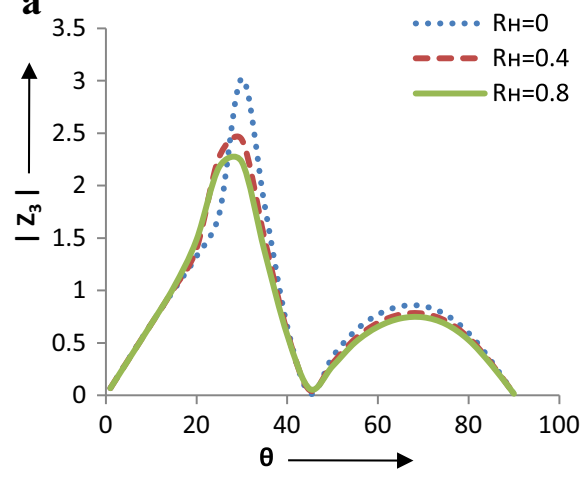

b

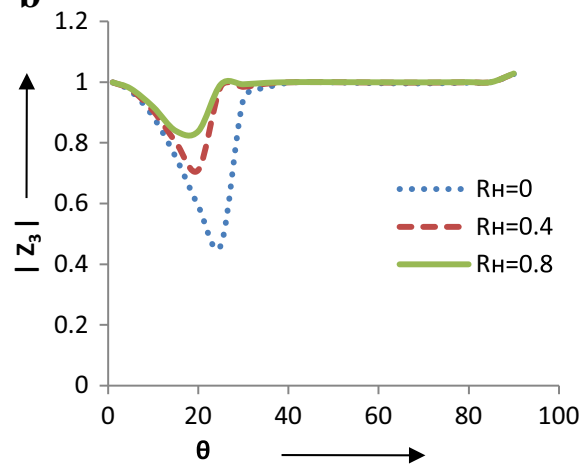

Fig. 7 Variation of the modulus of amplitude ratio of reflected $\mathrm{SV}$-wave $\left[\mathrm{Z}_{3}\right.$ ] from stress-free isothermal boundary with angle of incidence $(\theta)$ for different values magnetic field for a P-wave incidence and $\mathbf{b} \mathrm{SV}$-wave incidence
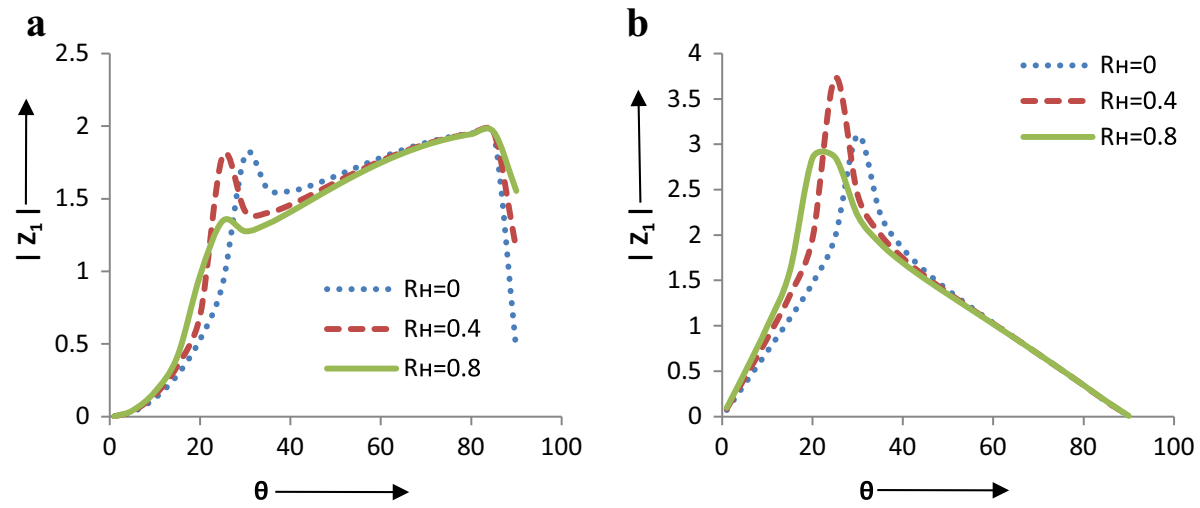

Fig. 8 Variation of the modulus of amplitude ratio of reflected thermal wave $\left[\mathrm{Z}_{1}\right]$ from rigid insulated boundary with angle of incidence $(\theta)$ for different values magnetic field for $\mathbf{a} \mathrm{P}$-wave incidence and $\mathbf{b} \mathrm{SV}$-wave incidence 

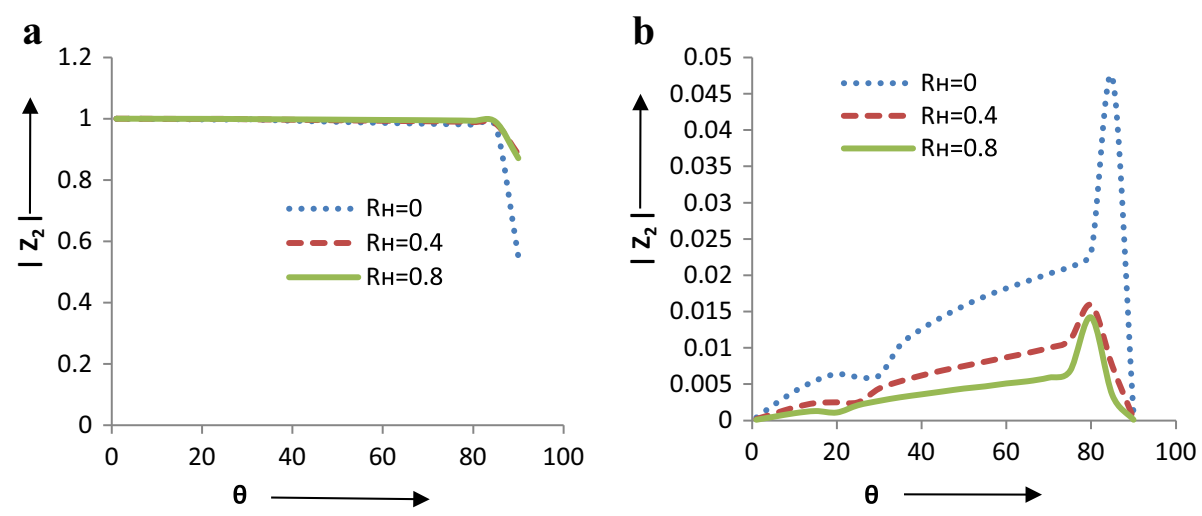

Fig. 9 Variation of the modulus of amplitude ratio of reflected P-wave [ $\left.\mathrm{Z}_{2}\right]$ from rigid insulated boundary with angle of incidence $(\theta)$ for different values magnetic field for $\mathbf{a} \mathrm{P}$-wave incidence and $\mathbf{b} \mathrm{SV}$-wave incidence

a

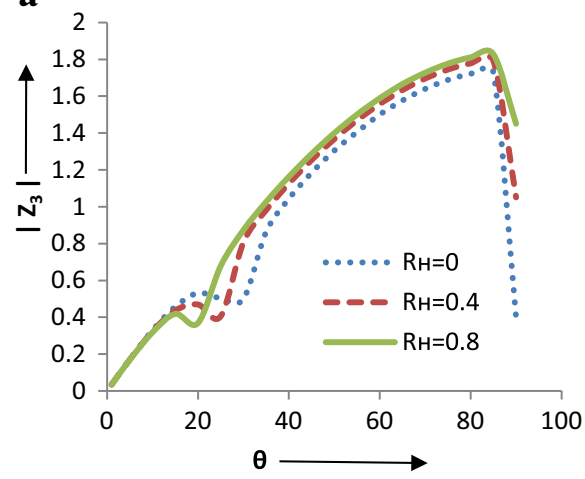

b

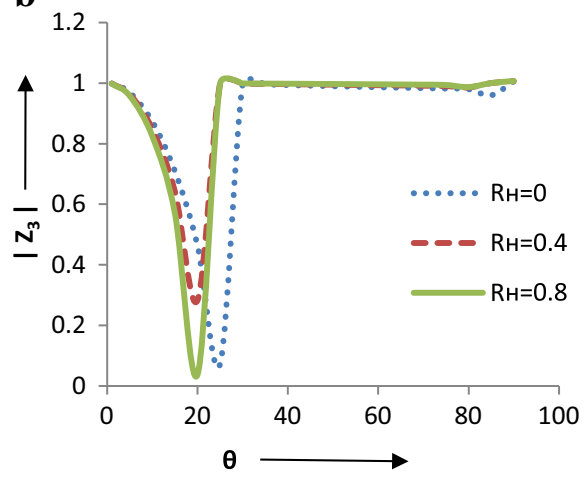

Fig. 10 Variation of the modulus of amplitude ratio of reflected SV-wave $\left[\mathrm{Z}_{3}\right]$ from rigid insulated boundary with angle of incidence $(\theta)$ for different values magnetic field for $\mathbf{a} \mathrm{P}$-wave incidence and $\mathbf{b} \mathrm{SV}$-wave incidence

For SV-wave incidence (Fig. 8b) on increasing the magnetic field the peak of $\left|\mathrm{z}_{1}\right|$ shifts to a lower angle of incidence.

Variation of $\left|z_{2}\right|$ versus $\theta$ curve for P-wave incidence remains unaffected by the application of magnetic field except after $\theta=85^{\circ}$ as seen from Fig. 9a. For SV-wave incidence, when magnetic field is applied, $\left|z_{2}\right|$ decreases and its peak shifts at a lower angle of incidence (Fig. 9b).

Figure 10a displays that on increasing the magnetic field, value of $\left|z_{3}\right|$ increases after $\theta=25^{\circ}$ and the notch on the curve of $\left|z_{3}\right|$ appears at a smaller value of $\theta$ for P-wave incidence. Figure 10b shows that with the application of magnetic field for SV-wave incidence, the dip of $\left|z_{3}\right|$ decreases and shifts to a lower value of $\theta$ and for further increase of magnetic field the dip reaches almost zero at the same value of $\theta$.

\section{Effect of Magnetic Field for Rigid Isothermal Boundary}

It is evident from Fig. 11a that with the application of magnetic field $\left|\mathrm{z}_{1}\right|$ starts from larger initial value and its peak shifts to a lower value of $\theta$ for P-wave incidence. For SV-wave 

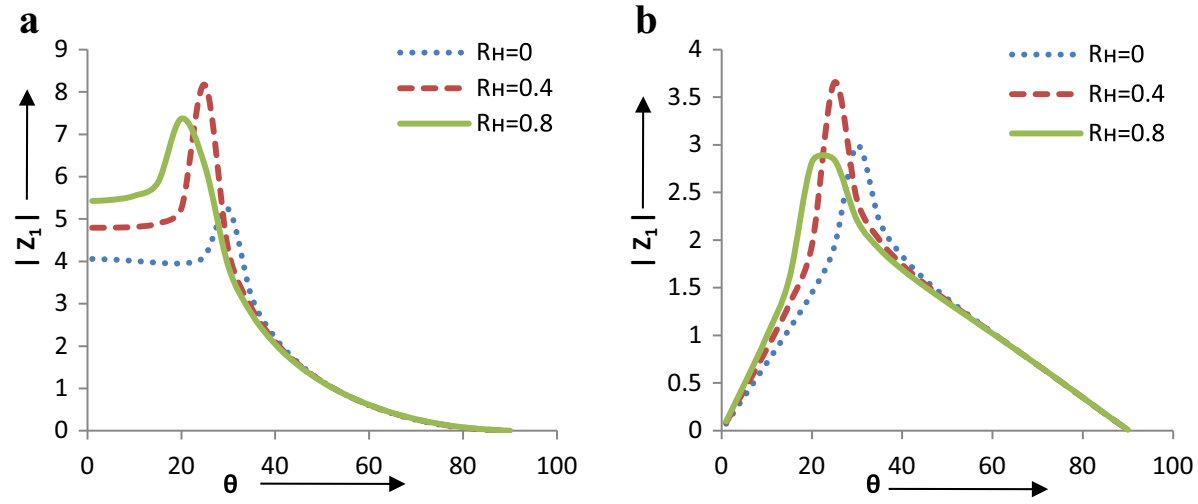

Fig. 11 Variation of the modulus of amplitude ratio of reflected thermal wave $\left[Z_{1}\right]$ from rigid isothermal boundary with angle of incidence $(\theta)$ for different values magnetic field for a P-wave incidence and $\mathbf{b} \mathrm{SV}$ wave incidence

a

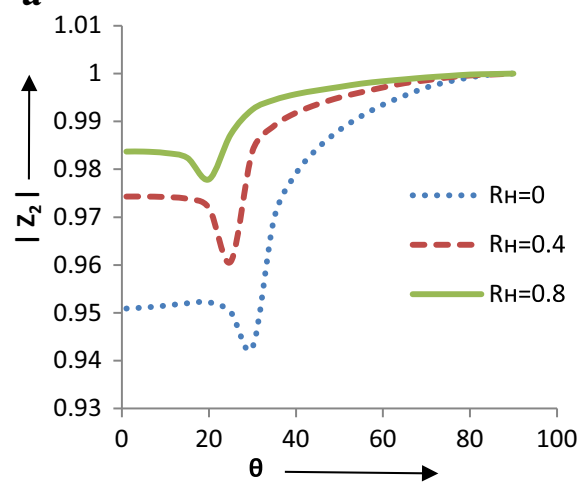

b

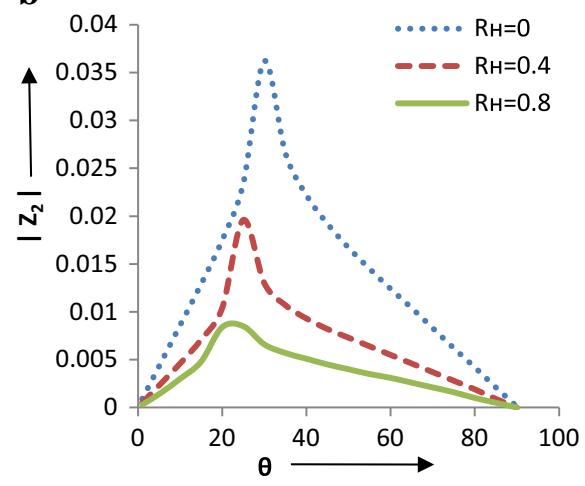

Fig. 12 Variation of the modulus of amplitude ratio of reflected $\mathrm{P}$-wave $\left[\mathrm{Z}_{2}\right]$ from rigid isothermal boundary with angle of incidence $(\theta)$ for different values magnetic field for $\mathbf{a} \mathrm{P}$-wave incidence and $\mathbf{b} \mathrm{SV}$-wave incidence

a

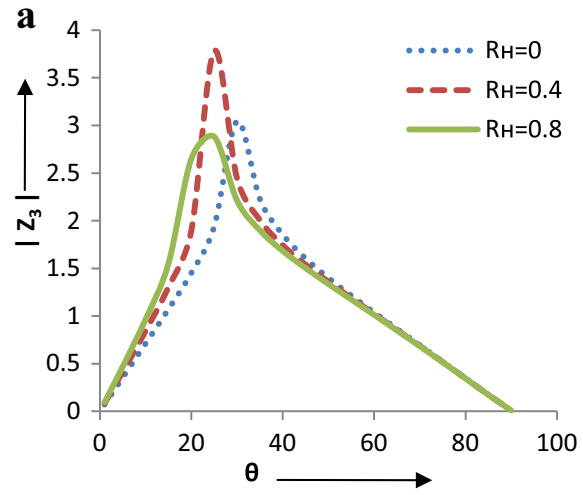

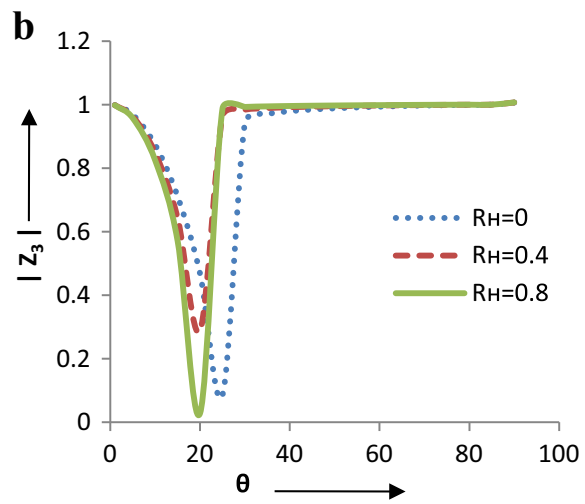

Fig. 13 Variation of the modulus of amplitude ratio of reflected $\mathrm{SV}$-wave $\left[\mathrm{Z}_{3}\right]$ from rigid isothermal boundary with angle of incidence $(\theta)$ for different values magnetic field for $\mathbf{a} \mathrm{P}$-wave incidence and $\mathbf{b} \mathrm{SV}$-wave incidence 
incidence (Fig. 11b), magnetic field shifts the peak of $\left|z_{1}\right|$ versus $\theta$ curve to a lower value of $\theta$.

Figure $12 \mathrm{a}$ shows that the initial value of $\left|\mathrm{z}_{2}\right|$ increases and the dip shifts to a smaller angle of incidence when magnetic field is increased for P-wave incidence. For SV-wave incidence, with the increase of the magnetic field, the value of $\left|z_{2}\right|$ (Fig. 12b) decreases and the maximum value occur at a lower angle of incidence.

For P-wave incidence, the peak of the curve of $\left|z_{3}\right|$ (Fig. 13a) occurs at a lower value of $\theta$ for larger values of magnetic field. For SV-wave incidence, the dip of curve of $\left|z_{3}\right|$ (Fig. 13b) decreases and shifts at a lower value $\theta$ and for further increase in magnetic field the dip increases at the same value of $\theta$.

\section{Conclusion}

Effect of magnetic field on the reflected components is more prominent for SV-wave incidence compared to P-wave incidence, when the boundary under consideration is an insulated (stressfree or rigid) one. However the effect of magnetic field brings significant changes in the result for isothermal (stress-free or rigid) boundaries irrespective of the nature of the incident wave. In case of stress-free boundaries, for SV-wave incidence the reflection coefficient curve of thermal wave and P-wave show a minimum (of nearly zero value) at $\theta=45^{\circ}$. Similarly for $\mathrm{P}$-wave incidence, the reflection coefficient curve of SV-wave shows a minimum at $\theta=45^{\circ}$ for the same stress-free boundary. These minima occur at the same value of angle of incidence $(\theta)$ even if the magnetic field is changed.

\section{References}

1. Biot, M.A.: Mechanics of Incremental Deformations. Wiley, New York (1965)

2. Lord, H.W., Shulmon, Y.: A generalized dynamical theory of thermoelasticity. J. Mech. Phys. Solids 15, 299-309 (1967)

3. Green, A.E., Lindsay, K.A.: Thermo elasticity. J. Elast. 2, 1-7 (1972)

4. Chandrasekharaiah, D.S.: Thermoelasticity with second sound: a review. Appl. Mech. Rev. 39, 355-376 (1986)

5. Green, A.E., Naghdi, P.M.: A re-examination of the basic postulates of thermo-mechanics. Proc. R. Soc. Lond. A Math. Phys. Sci. 432, 171-194 (1991)

6. Green, A.E., Naghdi, P.M.: On undamped heat waves in an elastic solid. J. Therm. Stress. 15, 253-264 (1992)

7. Green, A.E., Naghdi, P.M.: Thermo elasticity without energy dissipation. J. Elast. 31, 189-208 (1993)

8. Sinha, S.B., Elsibai, K.A.: Reflection of thermoelastic waves at a solid half-space with two relaxation times. J. Therm. Stress. 19, 749-762 (1996)

9. Sharma, J.N., Kumar, V., Chand, D.: Reflection of generalized thermoelastic waves from the boundary of a half-space. J. Therm. Stress. 26, 925-942 (2003)

10. Othman, M.I.A., Song, Y.: Reflection of plane waves from an elastic solid half-space under hydrostatic initial stress without energy dissipation. Int. J. Solids Struct. 44, 5651-5664 (2007)

11. Chakraborty, N., Singh, M.C.: Reflection and refraction of a plane thermoelastic wave at a solid-solid interface under perfect boundary condition, in presence of normal initial stress. Appl. Math. Model. 35, 5286-5301 (2011)

12. Singh, M.C., Chakraborty, N.: Reflection and refraction of P-, SV- and thermal wave, at an initially stressed solid-liquid interface in generalized thermoelasticity. Appl. Math. Model. 37, 463-475 (2013)

13. Knopoff, L.: The interaction between elastic wave motions and a magnetic field in electrical conductors. J. Geophys. Res. 60, 441-456 (1955)

14. Chadwick, P.: Elastic wave propagation in a magnetic field. In: Proceedings of the International Congress of Applied Mechanics, Brussels, Belgium (1957) 
15. Nowacki, W.: Dynamic Problems of Thermoelasticity. Noordhoff International Publishing, Leyden, The Netherlands (1975)

16. Paria, G.: On magneto-thermo-elastic plane waves. Math. Proc. Camb. Philos. Soc. 58, 527-531 (1962)

17. Roychoudhuri, S.K., Banerjee, S.: Magneto-thermoelastic waves induced by a thermal shock in a finitely conducting elastic half space. Int. J. Math. Math. Sci. 19, 131-144 (1996)

18. Singh, B.: Reflection of thermo-viscoelastic waves from free surface in the presence of magnetic field. Proc. Nat. Acad. Sci. India 72A, 109-120 (2002)

19. Roychoudhuri, S.K., Banerjee, M.: Magneto elastic plane waves in rotating media in thermo elasticity of type 11 (G. N. Model). Int. J. Math. Math. Sci. 71, 3917-3929 (2004)

20. Othman, M.I.A., Song, Y.: The effect of rotation on the reflection of magneto-thermoelastic waves under thermoelasticity without energy dissipation. Acta Mech. 184, 189-204 (2006)

21. Abo-Dahab, S.M., Salama, M.M.: A plane magnetothermoelastic waves reflection and refraction between two solid media with external heat sources and initial stress. J. Therm. Stress. 37, 1124-1151 (2014)

22. Singh, M.C., Chakraborty, N.: Reflection of a plane magneto-thermo-elastic wave at the boundary of a solid half-space in presence of initial stress. Appl. Math. Model. 39, 1409-1421 (2015) 